\title{
El sector hortofrutícola de Ecuador: Principales características socio-productivas de la red agroalimentaria de la uvilla (Physalis peruviana)
}

The fruit and vegetable sector in Ecuador: Main socio-productive characteristics of the agri-food network of Cape gooseberry (Physalis peruviana)

\author{
Carlos Moreno-Miranda ${ }^{1}$ \\ Raúl Moreno-Miranda ${ }^{2}$ \\ Araceli Alexandra Pilamala-Rosales ${ }^{3}$ \\ José Isaac Molina-Sánchez ${ }^{4}$ \\ Liliana Cerda-Mejía ${ }^{5}$
}

Fecha de recepción: 9 de septiembre de 2018

Fecha de aprobación: 2 de diciembre de 2018

\section{Resumen}

Si bien en Ecuador el sector de las frutas y hortalizas ha mostrado un crecimiento en mercados locales e internacionales, también ha enfrentado retos de carácter socioeconómico y productivo en su estructura y articulación que hasta el momento no han sido analizados; tal es el caso de la red agroalimentaria de la uvilla, situada en la zona andina central del país. En este sentido, el presente estudio muestra las principales características socio-productivas de la red agroalimentaria mencionada, la identificación de etapas, agentes y actividades (primarias y de soporte), y el análisis de los mecanismos que la gobiernan; para lo cual se empleó una metodología sistémica que permitió caracterizar la mencionada red, y en donde se determinó que presenta ventajas como la inclusión de mujeres a la etapa productiva, y el sentido de asociatividad de procesadores y distribuidores. Por otro lado, se concluyó que los productores requieren de formación en temáticas como poscosecha, logística de transporte, y calidad alimentaria. Asimismo, es necesario el fortalecimiento de estructuras asociativas que incentive una mayor participación de agentes en la etapa de producción. Finalmente, se dedujo que la red requiere aumentar el área asignada al cultivo de uvilla, y la aplicación de sistemas de producción bajo invernadero que incrementen los rendimientos, para de esa forma lograr ventajas en mercados internacionales.

Palabras clave: asociatividad; physalis peruviana; red; socio-productivo.

\footnotetext{
${ }^{1}$ M. Sc. Wageningen University (Wageningen, Países Bajos). carlos.morenomiranda@wur.nl. ORCID: 0000-0002-83787325.

${ }^{2}$ M. Sc. Universidad de Barcelona (Barcelona, España). rmorenmi7@alumnes.ub.edu.

${ }^{3}$ M. Sc. Universidad de Jaén (Jaén, España).

${ }^{4}$ M. Sc. Universidad Técnica de Ambato (Ambato, Ecuador). ji.molina@uta.edu.ec.

${ }^{5}$ Ph. D. Universidad Técnica de Ambato (Ambato, Ecuador). la.cerda@uta.edu.ec. ORCID: 0000-0002-5207-1869.
} 


\section{Abstract}

Although in Ecuador the fruit and vegetable sector has shown growth in local and international markets, it has also faced socio-economic and productive challenges in its structure and articulation that have not been analyzed so far; such is the case of the agrifood network of Cape gooseberry, located in the central Andean area of the country. In this sense, the present study shows the main socio-productive characteristics of the agrifood network mentioned, the identification of stages, agents and activities (primary and support), and the analysis of the mechanisms that govern it; for which a systemic methodology was used to characterize the aforementioned network, and where it was determined that it has advantages such as the inclusion of women in the productive stage, and the sense of associativity of processors and distributors. On the other hand, it was concluded that producers require training in topics such as post-harvest, transportation logistics, and food quality. Likewise, it is necessary to strengthen associative structures that encourage greater participation of agents in the production stage. Finally, it was deduced that the network requires to increase the cultivation area, and the application of greenhouse production systems to increase yields, with the purpose to achieve advantages in international markets.

Keywords: associative; network; physalis peruviana; socio-productive.

\section{Para citar este artículo:}

Moreno-Miranda, C., Moreno-Miranda, R., Pilamala-Rosales, A. A., Molina, J. I., CerdaMejía L. (2019). El sector hortofrutícola de Ecuador: Principales características socioproductivas de la red agroalimentaria de la uvilla (Physalis peruviana). Ciencia y Agricultura, 16(1), 31-51. DOI: https://doi.org/10.19053/01228420.v16.n1.2019.8809.

\section{Esta obra está bajo licencia internacional Creative Commons Reconocimiento 4.0}

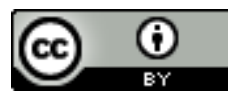




\section{Introducción}

Durante el período 2005 - 2012, los países como Perú, Chile, México, Ecuador, Costa Rica y Colombia presentaron una tendencia creciente en la producción de frutas y vegetales, lo que resultó en un incremento anual del $4,2 \%$ en las exportaciones hortofrutícolas de los mencionados países; en este evento también influyó el acontecimiento del 2011, donde los precios internacionales de metales y energía se redujeron $10,5 \%$ en promedio; ese comportamiento de los precios significó una lenta recuperación de las economías desarrolladas, y la desaceleración de economías emergentes sobre todo de China, quien se transformó en el principal importador de materias primas, y en el primer socio comercial de países latinoamericanos (FAO, 2011; CEPAL, FAO, IICA, 2015). Paralelamente a los mencionados acontecimientos del mercado internacional, las cadenas productivas de frutas y vegetales de algunos países principalmente centroamericanos experimentaron fuertes pérdidas en sus etapas y subsistemas de producción, como consecuencia de sequías, plagas y enfermedades, y condiciones climáticas; dificultando la producción de volúmenes de materias primas significativas que permitiesen enfrentar el mercado internacional, y ocasionando pérdidas en cierta medida del dinamismo de exportaciones agroalimentarias en América Latina (FAO, 2011; CEPAL, FAO, IICA, 2015).

Por otro lado, los países latinoamericanos presentan ventajas comparativas en el sector agropecuario; por lo tanto, sus esfuerzos deben consolidarse en ventajas competitivas que incorporen tecnología y sistemas de gestión eficientes a sus procesos productivos (Orjuela et al., 2008). Para ello, es necesario competir en función de una Red Productiva, la misma que va encaminada hacia implementar estructuras asociativas (Zambrano, 2016), capaces de definir estrategias que den respuestas integrales a los problemas del entorno interno y externo a la red, que canalicen eficientemente recursos, generen valor agregado, y logren el desarrollo sostenible de los agentes productivos del sector agroalimentario (Arras et al., 2017; Miranda, 2011; Scott, 2013).

El sector de frutas y hortalizas en el Ecuador ha mostrado un incremento en su participación tributando el $16 \%$ al PIB agrícola del país, esto sin considerar la producción de papas y banano (FAO, 2010; Glas et al., 2015). En el caso de 
explotaciones vegetales, las unidades de producción son relativamente pequeñas con superficies menores a las 100 ha y una alta diversificación de productos, asimismo se caracterizan por incidir frecuentemente en costos transaccionales (Blandon et al., 2008) debido a la falta de planificación en la oferta y la logística de transporte de materias primas, lo que evidencia las adversidades que enfrentan estos sistemas de producción (Paez, 2018). En la producción de frutas, las explotaciones son grandes (superficies mayores a las 100 ha), pero con un menor grado de diversificación de productos; estas presentan mecanismos de coordinación más eficientes en la logística de cosecha, clasificación y otros procedimientos que demandan los mercados nacionales como extranjeros (IFAD, 2011).

La producción de frutas y hortalizas constituye una alternativa económica viable para los sistemas familiares campesinos de producción o minifundistas, como es el caso de agricultores involucrados en la red productiva de la uvilla de la zona interandina del Ecuador donde se centró este estudio. Los cambios en la estructura que ha experimentado la mencionada red a través de la expansión de modernas unidades de producción, han significado una mejora en su desempeño de mercado (MAGAP, 2015). Pese a esto aún presenta retos que deben ser resueltos como la adaptación al dinamismo de mercados nacionales y extranjeros, adopción de modelos socio-productivos eficientes, encadenamientos productivos generadores de sinergias empresariales y la fundamentación de políticas agrarias de desarrollo (Hirschman, 1959). En este sentido, es necesario un estudio descriptivo de la estructura de la red productiva de la uvilla, considerando aspectos sociales y productivos, que aporte con información para la identificación de debilidades a nivel de red, permita establecer soluciones estratégicas integradoras; y mejore su desempeño económico y el bienestar de sus actores.

En la región Sierra Ecuatoriana, durante los últimos 5 años, el cultivo de uvilla se ha incrementado en un $10,0 \%$ siendo las provincias más sobresalientes Carchi, Imbabura, Cotopaxi, Tungurahua y Chimborazo, este incremento se genera gracias a las condiciones agroclimáticas que posee esta región, la planificación agrícola establecida por el estado, y el mejoramiento de variedades y 
productividad del cultivo liderado por el Instituto Nacional de Investigaciones Agropecuarias (MAGAP, 2017). Las oportunidades de mercado que poseen los productos exóticos en mercados europeos, norteamericanos y asiáticos también son determinantes que han motivado el aumento de área para el cultivo de este bien agrícola (Hilaca, 2017). Por otro lado, se calcula que existe una producción de 4.725,0 toneladas de uvilla por año, donde el 70,2\% es aprovechado por las agroindustrias y empresas exportadoras, mientras que el $29,8 \%$ es comercializado en estado fresco a nivel local y regional. En el mercado mundial, constantemente se genera el requerimiento de productos provenientes de cultivos no tradicionales como la uvilla, maracuyá, naranjilla, piña y tomate de árbol, lo que ha incentivado la diversificación de la producción y exportaciones en el Ecuador (PROECUADOR, 2016).

La uvilla ocupa un sitio importante dentro de los niveles de exportación de frutas. Al ser considerada una fruta exótica, es apetecida por muchas personas, alcanzando altos valores comerciales (Nuñez et al., 2014), además sus características nutricionales la hacen aún más atractiva para su mercado y comercialización (CORPEI-CICO, 2009). No obstante, a nivel nacional esta fruta es poco consumida debido al desconocimiento de los beneficios funcionales y nutricionales que posee; sin embargo, empresas farmacéuticas y biotecnológicas han manifestado las propiedades curativas-preventivas contra enfermedades prostáticas, cardiovasculares, digestivas y cancerígenas (ReyesBeltrán et al., 2017).

Según Tobar (2013), el Banco Central del Ecuador informó que durante el periodo 2007- 2012 ocurrió un incremento del 22,3\% en las exportaciones de uvilla con respecto al periodo 2001-2006, lo que significó 168,1 miles de dólares en valor FOB; sin embargo, el mayor competidor de Ecuador en las exportaciones de uvilla es Colombia en una relación 1 Ecuador: 15 Colombia. No obstante, desde enero 2017 Ecuador cuenta con la facilidad de ingreso al mercado de la Unión Europea ya que su oferta exportable no petrolera tiene libre acceso gracias al Acuerdo Comercial Multipartes que lo exonera de pagar el $3,6 \%$ de impuestos arancelarios sobre las exportaciones (Delegación de la Unión Europea en Ecuador, 2017). Por otro lado, durante el 2016 Ecuador logró 
incrementar $160,0 \%$ en sus exportaciones al mercado europeo de uvilla deshidratada (USD 782.000,0 valor FOB) con respecto al 2015 (USD 301.000,0 valor FOB), donde aspectos como responsabilidad social, precio justo y respeto del ambiente son claves para consolidar el mercado este producto (PROECUADOR, 2017).

La producción de uvilla no solo está destinada a la exportación de fruta en estado fresco, la misma que busca llegar al consumidor final con sus beneficios nutricionales intactos y forma original (Proaño, 2003); sino también es canalizada hacia su transformación en fruta congelada, puré, pulpa, mermeladas, conservas, y deshidratadas; siendo su objetivo la diversificación de consumo y expansión de mercado. Sin embargo, el mayor valor de mercado de uvilla ecuatoriana está formado por la fruta en estado fresco y los elaborados que logran mantener la forma geométrica de la fruta como es el caso de las conservas en lata o frasco de vidrio (Mendoza et al., 2012).

Por ello, la finalidad del estudio fue analizar de manera descriptiva la estructura de la red agroalimentaria de la uvilla considerando factores sociales y productivos; que permitiese la expansión del conocimiento de la mencionada red, y aporte con información para la identificación de potenciales problemas que requieran de soluciones estratégicas; esto se logró mediante la identificación de agentes de la red productiva de la uvilla en la zona interandina central, el análisis de factores socio-productivos de los diferentes eslabones, y la identificación del mecanismo que dinamiza y gobierna la red productiva bajo estudio.

\section{Materiales y métodos}

El estudio se realizó en la zona Interandina comprendida por las provincias de Cotopaxi, Tungurahua y Chimborazo (extensión aproximada de 59810 km²). Esta se localiza geográficamente a $0^{\circ} 42^{\prime}$ latitud sur y $80^{\circ} 00^{\prime}$ longitud oeste, con una altitud promedio de $3500 \mathrm{msnm}$, una temperatura promedio que oscila entre 15 y $25^{\circ} \mathrm{C}$. 


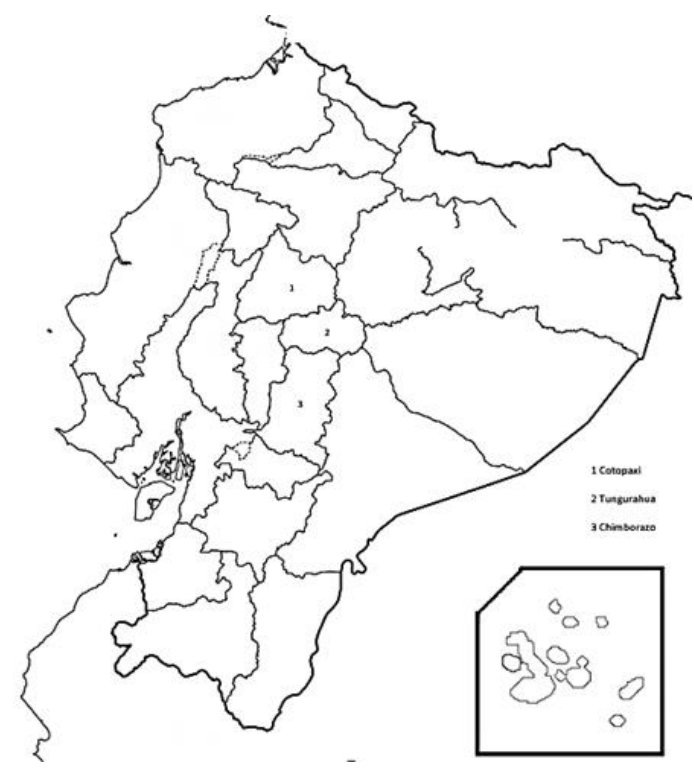

Fig. 1. Mapa del territorio ecuatoriano y zona comprendida por las provincias Cotopaxi, Tungurahua y Chimborazo (SENPLADES, 2018).

En la zona se aplicó una metodología sistémica que buscó detectar los principales flujos de recursos a lo largo de la red productiva bajo estudio; así como, analizar las características socio-productivas más relevantes de cada etapa de la red, identificar tendencias sociales, productivas y vinculatorias entre los diferentes agentes, como se detalla a continuación:

\section{A. Identificación de agentes que conforman las etapas de la red productiva}

Se consideró la información del último censo del 2013 generado por el Ministerio de Agricultura, Ganadería y Pesca (MAGAP), y el registro de empresas involucradas en la cadena de valor de la uvilla proporcionado por el Ministerio de industrias y Productividad (MIPRO). Se aplicó el esquema de cadena de valor de Porter, agrupando agentes por etapas.

\section{B. Descripción del tamaño de la muestra}

Para la determinación del tamaño de la muestra a nivel productor se utilizó la variable continua "Número de productores de uvilla registrados por el MAGAP" de las provincias involucradas, misma que representa un total de 434 productores (población); para ello, se empleó la fórmula reportada por Sukhatme (1956) con un nivel de confianza del 90,0\% obteniéndose como resultado una muestra mínima de 120 productores. La muestra de productores de uvilla se 
distribuyó de la siguiente manera: 36 en la provincia de Cotopaxi, 55 en Tungurahua y 37 en Chimborazo logrando una representatividad del $30,0 \%$ de la población total. Mientras que para la etapa de posproducción se usó la información de empresas participantes registradas en el Ministerio de Industrias y Productividad.

\section{Análisis de aspectos socio-productivos}

Se realizó a través de la aplicación de encuestas y entrevistas estructuradas que contemplaron variables de carácter productivo y socioeconómico, obteniéndose un diagnóstico de la situación actual de la red productiva de la uvilla.

\section{Dinámica de gobernanza de la red productiva}

Se identificaron los mecanismos que gobiernan la red productiva en base a la información recabada de los agentes y mediante la aplicación del método propuesto por Gereffi \& Stacey (2009), el cual reconoce los eslabones que ejercen gobernanza, liderazgo o control de una cadena o red, asimismo resaltar el valor agregado que se genera en las distintas etapas y finalmente reconocer los eslabones más idóneos para el escalamiento económico mediante estrategias.

\section{Resultados y discusión}

Los resultados del estudio inician con la descripción de los agentes identificados en las diferentes etapas de la cadena de valor de la uvilla, además presentan un esquema estructural de las diferentes conexiones y grado de coordinación. Por otro lado, se describe los diferentes aspectos socio-productivos de los principales eslabones de la cadena productiva, siendo estos: producción, procesamiento y distribución.

\section{A. Identificación de agentes que conforman las etapas de la cadena de valor}

La evaluación de los objetivos y recursos de las empresas dedicadas a la transformación de materia prima en producto terminado permite identificar las competencias industriales como: la amenaza de los nuevos competidores, rivalidad entre competidores, poder de negociación de los proveedores, poder 
de negociación de los clientes, y amenazas de servicios y productos sustitutos (Herrera \& Baquero, 2018)

La red productiva de la uvilla de la zona bajo estudio se caracteriza porque el poder de negociación de sus productores es alto, debido a que la cantidad de participantes es relativamente pequeña (menos de 500 participantes) en relación al número de productores de redes extensas (más de 500 participantes) (Alderete, 2015), como son las redes de mora, ajo, hortalizas y leguminosas; posiblemente esto se debe a que la demanda de uvilla por parte de la industria y el consumidor final es aún pequeña, por tanto incentiva a pocos agentes a su cultivo. Por otro lado, la zona Interandina del Ecuador por sus características territoriales, capacidades y dinámicas poblacionales, es considerada un importante nodo comercial a escala nacional lo que significa una importante ventaja competitiva para los agentes de la red bajo estudio.

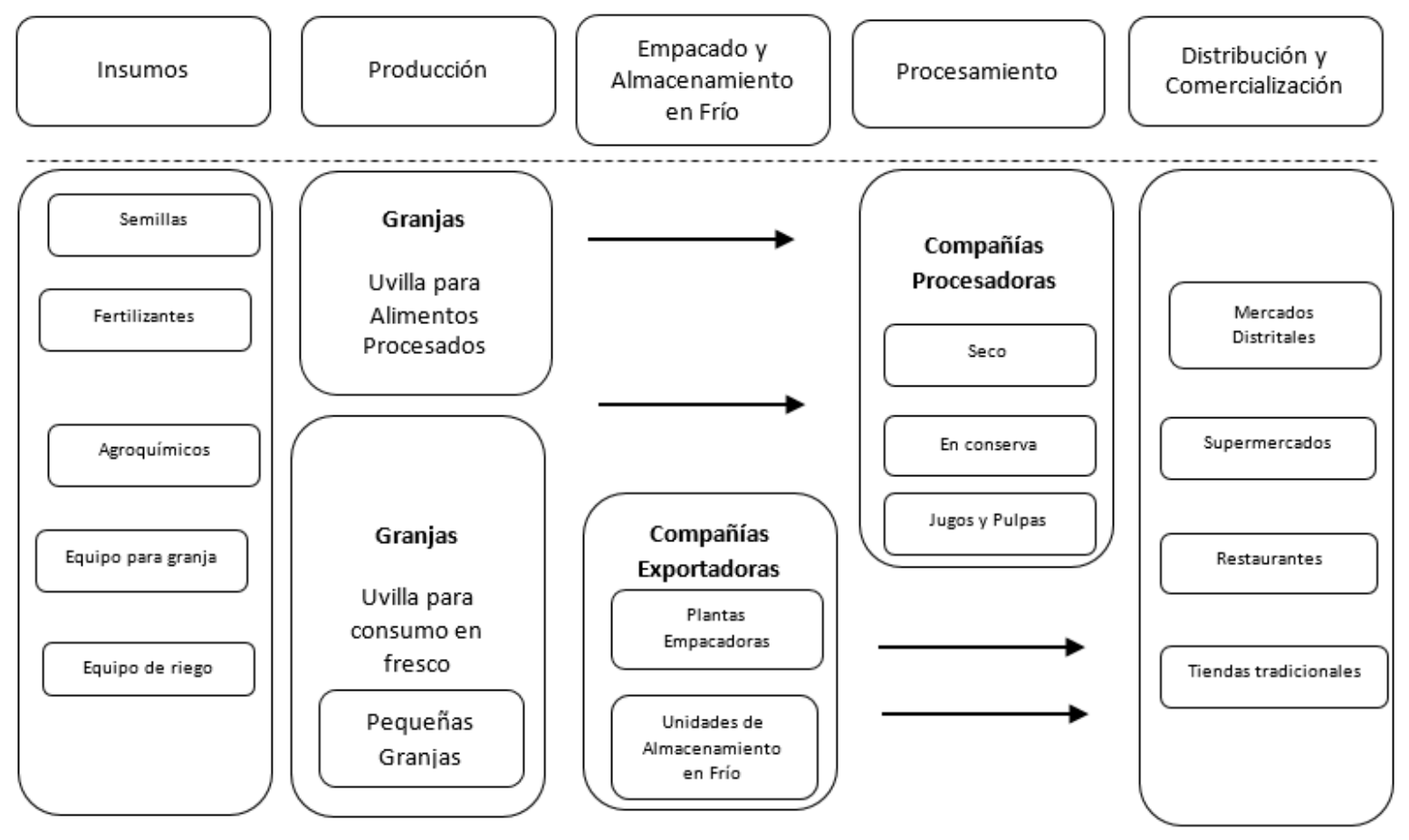

Fig. 2. Agentes de la red productiva de la uvilla.

La Figura 2, esquematiza la red productiva con su punto de partida en actividades de soporte, llevadas a cabo por agentes como empresas productoras estatales y privadas, que suministran a través de flujos de insumos semillas, maquinaria, fertilizantes, entre otros bienes; de igual forma participan 
El sector hortofrutícola de Ecuador: Principales características socio-productivas de la red agroalimentaria de la uvilla (Physalis peruviana)

cooperativas de ahorro y crédito, y el banco estatal, quienes son los encargados de financiar las diferentes actividades a lo largo de la red productiva.

De acuerdo con la información proporcionada por el MAGAP presentada en la Tabla № 1, se identificó que para la etapa de producción de uvilla de la zona comprendida por las provincias de Chimborazo, Cotopaxi y Tungurahua se cuenta con el $0,8 \%$ de productores del sector hortofrutícola. A la vez, la provincia de Tungurahua se destaca por poseer el mayor número de hectáreas para la producción de uvilla. Es importante mencionar que existe una ventaja significativa en cuanto a la cosecha de esta fruta, ya que es recolectada durante todos los días del año, por lo que se pueden obtener grandes volúmenes de producción (Coronel, 2016).

Tabla 1. Número de productores de uvilla y superficie de producción de uvilla en la zona centro de Ecuador MAGAP (2015).

\begin{tabular}{|c|c|c|}
\hline Provincia & $\begin{array}{c}\text { Número de productores de uvilla } \\
\text { registrados por el MAGAP }\end{array}$ & $\begin{array}{c}\text { Superficie (hectáreas) de producción de } \\
\text { uvilla registradas por el MAGAP }\end{array}$ \\
\hline Cotopaxi & 123 & 258,6 \\
\hline Tungurahua & 177 & 325,2 \\
\hline Chimborazo & 134 & 271,5 \\
\hline
\end{tabular}

La etapa de producción; es ejecutada por agricultores individuales, asociaciones y/o cooperativas agrícolas; estos agentes direccionan su producción hacia intermediarios, centros de distribución provinciales, mercados distritales, y procesadoras privadas de frutas y vegetales. Los procesadores y agentes encargados de actividades de poscosecha direccionan la producción hacia brokers, mayoristas, minoristas y mercados institucionales en Cadenas Hoteleras, Restaurantes y Servicios de Caterin (HORECA), quienes son los encargados de proveer de los bienes generados en la red al consumidor final.

Para la etapa de posproducción, se identificaron 23 empresas registradas en el Ministerio de Industrias y Productividad, quien es el ente gubernamental encargado de caracterizar las diferentes mipymes y grandes empresas basándose en la actividad y el sector productivo donde intervienen. Las empresas son registradas de acuerdo a la actividad principal que ejecutan dentro de la cadena productiva, como se explica en la Figura 3. 


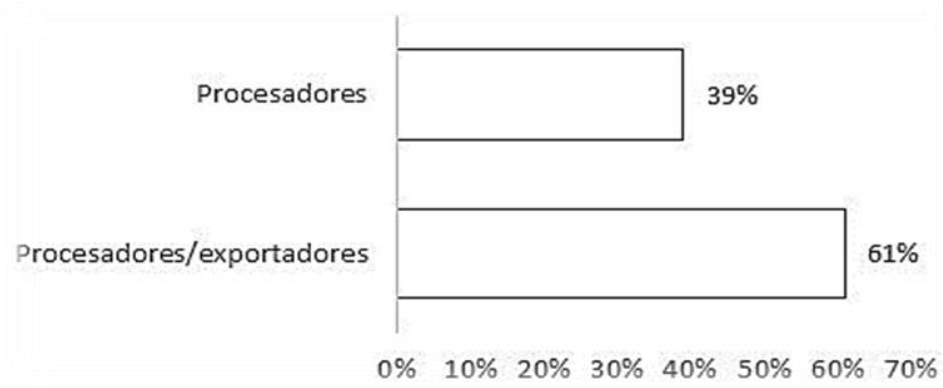

Fig. 3. Distribución de las funciones de las empresas.

La información recabada permitió identificar que el 78,3\% de las empresas encuestadas incluye uvilla en su cartera de negocios, ya sea en estado fresco o procesado. Solo el $21,7 \%$ de las empresas transforma uvilla en productos como jugos, pulpa, zumo, almíbares y deshidratada. Asimismo, se identificó que el $39,1 \%$ de las empresas registradas operan exclusivamente en el mercado doméstico de uvilla, el 5,0\% exclusivamente en el mercado internacional; y el $55,9 \%$ ha participado en ambos tipos de mercado como se muestra en la Figura 4. Por otro lado, del 60,9\% de empresas que ha tenido experiencia en mercados internacionales, solo el $30,4 \%$ ha exportado productos a base de uvilla con valor agregado.

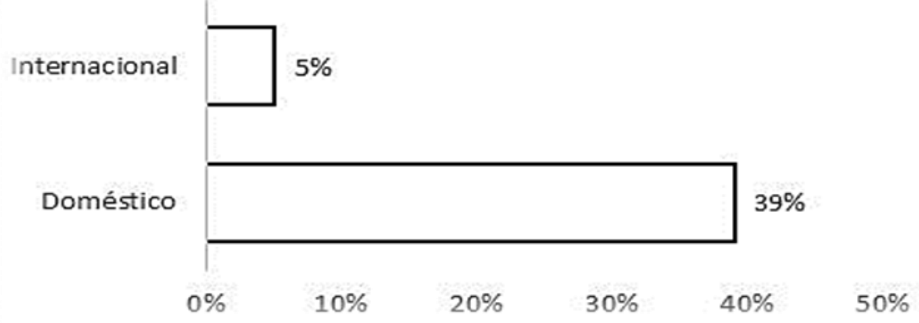

Fig. 4. Participación de empresas privadas en el mercado de uvilla.

A pesar del bajo porcentaje de transformadores de uvilla $(21,7 \%)$ en productos convencionales (jugos, conservas y deshidratados), existen varios componentes derivados de esta fruta que pueden ser aprovechados para la fabricación de bienes alimenticios de mayor valor, como es el caso de bioactivos (vitaminas, antioxidantes) que esta fruta posee. Illesca (2017), afirma mediante un estudio realizado en empresas del cantón Ambato, provincia de Tungurahua, que el $23,0 \%$ comercializa uvilla como fruto fresco, 12,0\% como mermelada, $9,0 \%$ destina a la transformación en jugo; pero el 56,0\% de las empresas encuestadas 
no mostró interés alguno en transformar uvilla en bienes de consumo; esto debido principalmente al desconocimiento de beneficios económicos, productivos y nutricionales.

Con respecto a los distribuidores registrados por el Ministerio de Industrias y Productividad, se identificaron tres corporaciones que lideran el mercado minorista de uvilla en estado fresco y procesado; mientras que los distribuidores restantes (10 identificados) son empresas medianas de actividad comercial mayorista

\section{B. Análisis de aspectos socio-productivos - Producción}

Esta sección presenta un análisis de los principales aspectos de índole socioeconómico y productivo, que caracteriza a la etapa de producción de la cadena de valor de la uvilla de la zona interandina central de Ecuador.

1) Factor socioeconómico. Esta etapa cuenta con productores entre los 30 y 50 años, en su mayoría son hombres $(56,0 \%)$ pero la inclusión de mujeres se ha incrementado en un 5,0\% en los últimos 3 años gracias al aumento progresivo del mercado de uvilla. La formación académica de sus agentes es secundaria $(40,0 \%)$ y superior $(26,0 \%)$. Un $28 \%$ de los productores son miembros de asociaciones y un $39,0 \%$ son socios de cooperativas. Con lo que respecta a sus fuentes de financiamiento, estas varían como lo muestra la Figura 5, donde es el capital propio el que mayormente interviene en la producción y lo que posiblemente impida un crecimiento económico rápido de este bien agroalimentario.

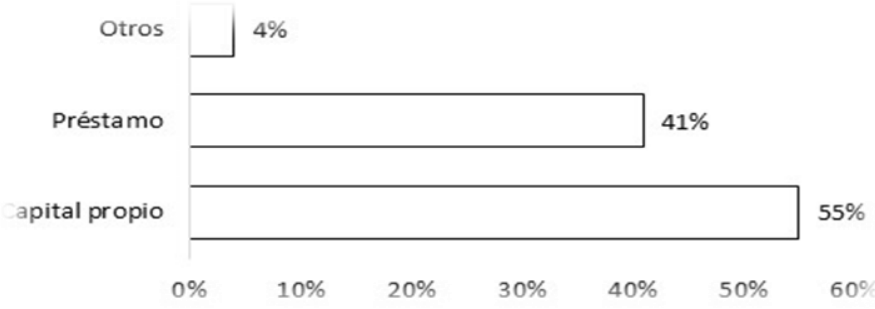

Fig. 5. Fuentes de financiamiento de los productores de uvilla. 
Según Brito, D. citado por Villacrés. G (2012), para la producción de un cultivo de 60 hectáreas de uvilla se requiere aproximadamente de USD 6.000,0. Los agricultores de uvilla de las provincias de Tungurahua y Cotopaxi, registran créditos de USD14.500,0 en promedio para la inversión en la producción del mencionado producto agrícola (Banco Nacional del Fomento, 2012). Se considera como una ventaja que los costos de producción de uvilla en los países andinos son relativamente bajos, mientras que los valores por ingresos de las exportaciones de uvilla superan cinco veces los costos de producción, generándose así márgenes financieros muy atractivos para la industria y por tanto para el país. Guerrero (2012), indica que el precio para el mercado doméstico de la uvilla fresca con capuchón (cáliz de la flor) es de USD 1,0 / Kg y el precio de la uvilla sin capuchón es de USD 1,5/Kg; mientras que para el mercado internacional el precio de la uvilla fresca con capuchón oscila entre USD 5,0 y $9,0 / \mathrm{Kg}$ considerando que en estos valores se incluye el costo del transporte, tarifas arancelarias y seguros de carga (Almeida, 2012).

2) Factor productivo. En cuanto a tenencia de tierra, un $45 \%$ posee tierras propias, $29 \%$ de los encuestados labora en sociedad y un $26 \%$ arrienda tierras y las dedica a I agricultura. El 55\% de los encuestados posee una extensión de tierra mayor a 3 ha, y el área de cultivo para uvilla va entre 0,1 a 1,5 ha en promedio. A la vez, el 56\% aplica técnicas de cultivo a campo abierto.

El genotipo mayormente cultivado es el ecuatoriano, seguido del genotipo colombiano y en menor proporción el keniano (46\%, 35\% y 19\% respectivamente). Según Brito citado por Altamirano M. (2010) existen cerca de 20 ecotipos (genotipos adaptados) de uvilla que se diferencian principalmente por el color y tamaño. Además, menciona que en Ecuador hay un ecotipo anaranjado, adaptado a las condiciones agroclimáticas ecuatorianas, de tamaño mediano a grande y que es comercializado en el mercado nacional e internacional.

Rodríguez y Bueno reportado por Fischer et al., (2014), manifiestan que Colombia siendo el mayor productor de uvilla en el mundo cultiva mayormente el 
El sector hortofrutícola de Ecuador: Principales características socio-productivas de la red agroalimentaria de la uvilla (Physalis peruviana)

genotipo colombiano $(2 n=32)$ y en menor proporción el genotipo keniano $(2 n=48)$.

De acuerdo con los resultados y comparándolos con los rendimientos reportados por Altamirano (2010) y Fischer, Almanza-Merchán y Miranda (2014) donde mencionan que el rendimiento de uvilla para Ecuador es de 13,6 ton/ha y para Colombia de 14,5 ton/ha, se observa que la cadena de valor bajo estudio presenta valores muy similares en esta variable. Adicionalmente, es importante mencionar que solo un 33\% de los productores aplican técnicas de poscosecha que consisten en: i) distribución de la cosecha en contenedores plásticos de 10 kg de capacidad para su transporte a bodegas o centros de acopio, ii) selección de la fruta por tamaño (calibre) y estado de madurez. El porcentaje de quienes aplican técnicas de poscosecha es relativamente bajo debido a varios factores como falta de conocimiento técnico, poca exigencia de calidad en mercados locales e interés; y escaza intervención por parte del sector académico en esta red productiva.

\section{Análisis de aspectos socio-productivos - Posproducción: Procesadores}

En esta sección se presenta un análisis principalmente de índole socioproductivo de la etapa de posproducción que hemos denominado "procesadores", y que está operada por empresas encargadas de la transformación de la uvilla que proviene de la zona interandina central de Ecuador.

1) Factor socioeconómico. De las empresas registradas se identificó que más del $70 \%$ corresponde a Mipymes (micro y pequeñas empresas); asimismo, se identificó medianas y grandes empresas participando en la cadena de valor de la uvilla como lo muestra la Figura 6 . El $64 \%$ de las empresas registradas son miembros de asociaciones y un $36 \%$ son socios de cooperativas. 


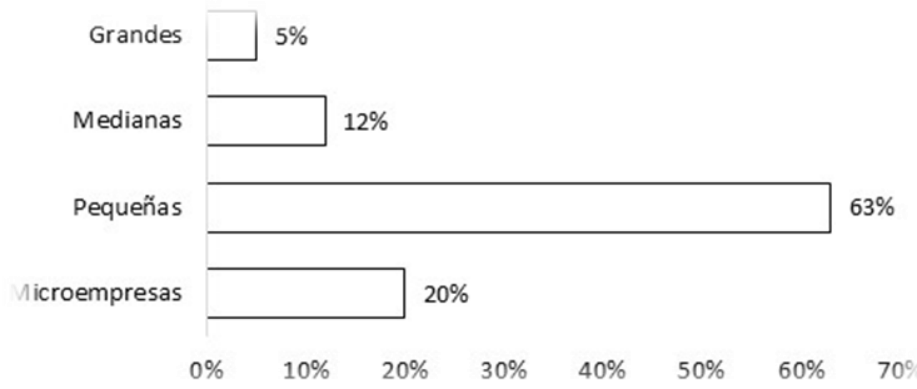

Fig. 6. Tipos de empresas participantes en la cadena de valor de la uvilla.

Por otro lado, con respecto al financiamiento un $37 \%$ de los procesadores opera mayormente con recursos propios y deuda, mientras que un $41 \%$ lo ejecuta mediante deudas y acciones en la bolsa. Esto que indica una importante intervención del capital de terceros como cooperativas de ahorro y crédito, entidades bancarias públicas y privadas; e inversionistas. Asimismo, se observa que el procesador también adopta cierto porcentaje del riesgo financiero que implica la actividad productiva como la muestra la Figura 7.

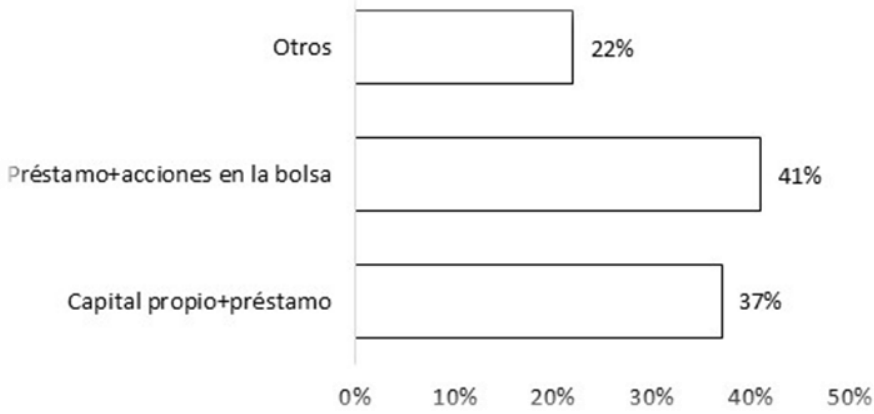

Fig. 7. Fuentes de financiamiento de los procesadores de uvilla.

Un dato relevante fue que el $57 \%$ de las empresas no lleva a cabo la compra de fruta cuando esta es de baja calidad ya sea por daños mecánicos, calibre pequeño, daños por insectos, presencia de moho entre otras razones, esto debido a que lo perciben como un riesgo que va en desmedro de la calidad final de producto procesado principalmente de fruta deshidratada y en almíbar. Por otro lado, se estableció que un $43 \%$ de los procesadores comercializan uvilla en segunda y cuarta gamma, y su entrega la realizan en mercados exclusivamente internacionales (36\%) y en mercados exclusivamente nacionales (49\%); el 15\% restante de procesadores lo hace en ambos formatos. Adicionalmente, se 
El sector hortofrutícola de Ecuador: Principales características socio-productivas de la red agroalimentaria de la uvilla (Physalis peruviana)

observó que los procesadores se rigen a controles sanitarios minucioso ejecutados por la Agencia Nacional de Regulación, Control y Vigilancia Sanitaria (ARCSA) durante todo el proceso de trasformación de la fruta, lo que genera costos adicionales que incrementan el precio final de venta al consumidor (Montilla et al., 2016).

Por otro lado, es posible que la comercialización de uvilla no solo se realice como fruta fresca, sino que existe un sin número de productos que pueden ser comercializados en el mercado nacional e internacional, tal y como lo indica Andrade (2012), quien menciona que productos como yogurt, néctar, almíbar y chocolates rellenos son los mayormente fabricados y utilizan como ingrediente principal uvilla.

\section{Análisis de aspectos socio-productivos - Posproducción: Distribuidores}

En esta sección se presenta un análisis principalmente de índole socioproductivo de la etapa de posproducción que hemos denominado "distribuidores", y que está operada por empresas encargadas de la distribución de bienes de consumo a base de uvilla cultivada en la zona interandina central de Ecuador.

1) Factor socioeconómico. Entre los distribuidores se identificó que el $50 \%$ son Mipymes y el 50\% restante son grandes empresas. El 50\% de los distribuidores son miembros de asociaciones y el $100 \%$ no pertenecen a cooperativas. Por otro lado, con respecto al financiamiento un $72 \%$ de ellas operan mayormente con recursos propios y deuda, y un $28 \%$ lo ejecuta mediante deudas y acciones en la bolsa de valores.

La comercialización de productos de uvilla es ejecutada por distribuidores mayoristas y minoristas; por un lado los minoristas (retailers) canalizan los productos hacia el consumidor final (Equipo OCE Nueva York, 2018), mientras que los mayoristas son los encargos de la logística que implica la exportación de los productos de uvilla, y son quienes acuerdan precios, periodos de suministro, condiciones de pago, entre otros aspectos (Hinojosa \& Ipiales, 2012). Cabe mencionar que el principal producto de uvilla ecuatoriana comercializado a nivel 
internacional es el deshidratado, esto debido a que el ítem conserva sus propiedades nutricionales y vitamínicas al ser secado a menos de $60^{\circ} \mathrm{C}$. Es importante recalcar que el Equipo OCE Nueva York (2018) menciona que para el año 2020 se espera que el consumo mundial de frutas deshidratas crezca alrededor de 4 millones de toneladas de los cuales el 10\% sea tributado por uvilla y otro tipo de berries. En cuanto a las exportaciones para los Estados Unidos de fruta deshidratada, Ecuador se encuentra en el puesto 19 con una participación del $1 \%$.

Por otro lado, Ecuador ha creado alianzas de exportación con La Unión Europea, siendo Alemania el mercado objetivo para la distribución de estos productos (Herrera, 2015). Un claro ejemplo de las exportaciones es la asociación Sumak Mikuy quien no requiere de un intermediario para su distribución, en vista de que su producto fue introducido al mercado alemán con una exportación de 500 kilogramos de uvilla orgánica. Así también se evidencio que en el periodo 20112015 hubo una tasa de crecimiento promedio anual del 3,7\% y $12,3 \%$ en valor FOB y en volumen respectivamente (El productor, 2017).

\section{E. Dinámica de gobernanza de la cadena de valor de la uvilla}

El análisis de gobernabilidad permite comprender cómo se controla y coordina una cadena cuando ciertos actores de la cadena tienen más poder que otros. Gereffi (1994) definió la gobernabilidad como "relaciones de autoridad y poder que determinan cómo los recursos financieros, materiales y humanos se asignan y fluyen dentro de una cadena.

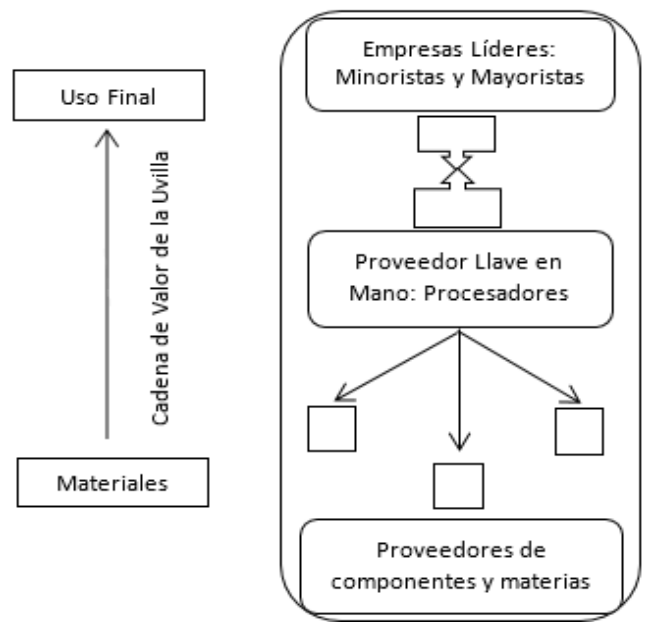

Fig. 8. Dinámica de gobernanza de la red productiva de la uvilla en Ecuador. 
La Figura 8, esquematiza la dinámica de gobernanza de la red productiva de la uvilla en Ecuador. En este esquema la complejidad de la información compartida entre los actores de la cadena, la codificación de dicha información y el nivel de competencia del proveedor son esclarecidos. Según la clasificación propuesta por Frederick \& Gereffi, 2009; Gereffi et al., 2005, la dinámica de gobierno identificada es la modular, debido a que las transacciones son relativamente fáciles de codificar. Del mismo modo, los procesadores fabrican bienes de consumo según las especificaciones de mayoristas y minoristas, asumiendo la responsabilidad total de la tecnología utilizada para procesamiento y empleando maquinaria genérica que diluye las inversiones en una amplia base de clientes. Esto mantiene los costos de cambio bajos y limita las inversiones específicas en tecnología. Los vínculos son más importantes que en los mercados simples debido al alto volumen de información que fluye a través del vínculo entre empresas. La tecnología de la información y el intercambio de información son cruciales para el funcionamiento de las cadenas de valor agroalimentarias con un gobierno modular.

\section{Conclusiones}

En la red productiva de la uvilla ecuatoriana, se involucran instituciones públicas y privadas, quienes participan principalmente en la etapa de dotación de recursos (semillas, fertilizantes, agroquímicos, capital, entre otros) como actividad de soporte; esta etapa se constituye en el inicio del flujo de recursos tangibles necesarios para el desempeño de esta red productiva.

La etapa de producción cuenta con características sociodemográficas favorables, como la intervención de participantes relativamente jóvenes cuya edad oscila entre los 30 y 40 años, y la inclusión de mujeres a la red productiva en los últimos 3 años gracias a las oportunidades de mercado de la uvilla; pero asimismo, la red productiva de la uvilla presenta aspectos que requieren atención; por ejemplo, la formación del conocimiento técnico de sus productores en temáticas como poscosecha, logística de transporte, y calidad alimentaria; y el fortalecimiento de estructuras asociativas en función de beneficios tangibles, que incentive una mayor participación de los productores en la red. Un aspecto 
relevante que también necesita atención es el productivo; debido a que se requiere del aumento significativo del área asignado al cultivo de uvilla, y la aplicación de sistemas de producción bajo invernadero que permitan incrementar los rendimientos.

La red productiva de la uvilla en la zona interandina presenta una etapa de posproducción que define claramente el accionar de procesadores y distribuidores. Pequeñas empresas son mayormente quienes participan en las actividades de valor agregado $\mathrm{y}$, presentan un porcentaje muy importante de pertenencia a asociaciones. Por otro lado, las empresas distribuidoras también presentan un importante sentido de asociatividad, pero se caracterizan por el modelo de negocios con el que operan, por ejemplo, participando en la bolsa de valores, implementando métodos de negociación bajo crédito que generen flujos de efectivo positivos.

\section{Referencias}

Alderete, M. (2015). Redes de Pymes: Una visión desde las teorías de club y de equipo. Revista de Economía Institucional, 17(32), 317-348. DOI: https://doi.org/10.18601/01245996.v17n32.11.

Almeida, D. F. (2012). Estudio de factibilidad para la creación de una empresa dedicada a la comercialización de la uvilla y sus derivados para la comunidad Europea. Tesis de grado, Universidad Internacional del Ecuador, Ecuador.

Altamirano, M. (2010). Estudio de la cadena productiva de uvilla (Physalis peruviana L.) en la Sierra norte del Ecuador. Tesis de grado, Universidad San Francisco de Quito, Ecuador.

Andrade, M. V. (2012). Diseño de una planta para la obtención de cuatro productos a base de uvilla (Physalis peruviana) en la provincia de Pichincha. Tesis de maestría, Universidad de Las Américas, Ecuador.

Arras, A., Fierro, L., Jáquez, J., \& López, J. (2017). Enterprise strategy in the insertion to value chain. Revista Mexicana de Agronegocios, 26, 215-225.

Banco Nacional del Fomento (2012). Gerencia de credito y estadistica. Recuperado de: https://contenido.bce.fin.ec/documentos/PublicacionesNotas/Catalogo/Anuario/80anios/Notas185anios.pdf.

Blandon, J., Henson, S., \& Cranfield, J. (2008). Small-Scale Farmer Participation in New Afri-Food Supply Chains: Case of the Supermarket Supply Chain for Fruit and Vegetables in Honduras. The International Journal of Developmental, 96, 971-984. DOI: https://doi.org/10.1002/iid.1490.

CEPAL, FAO, IICA (2015). Instituto Interamericano de Cooperación para la Agricultura. Recuperado de: http://www.iica.int/sites/default/files/publications/files/2015/b3695e.pdf.

Coronel, P. A. (2016). Plan de negocios para la " Creación de una empresa dedicada a la fabricación y comercialización de mermeladas hechas a base de uvillas en la ciudad de Quito". Tesis de Maestría, Universidad de las Américas, Ecuador.

CORPEI - CICO (2009). Corporación de Promoción de Exportaciones e Inversiones. Centro de Información e Inteligencia Comercial. Recuperado de: http://www.pucesi.edu.ec/pdf/uvilla.pdf.

Delegación de la Unión Europea en Ecuador (2017). European External Action Service. Recuperado de: https://eeas.europa.eu/sites/eeas/files/20150505 triptico acuerdo ue ecuador es.pdf.

El productor (2017). Reporte Técnico. Ecuador: Incrementa la exportación de uvilla deshidratada hacia Alemania en un 160\%. Recuperado de: http://elproductor.com/noticias/ecuador-incrementa-la-exportacion-de-uvilla-deshidratadahacia-alemania-en-un-160.

Equipo OCE Nueva York (2018). Mango, piña, uvilla y banana deshidratada en el mercado de los Estados Unidos Marzo 2018. Recuperado de: https://www.proecuador.gob.ec/mango-pina-uvilla-y-banana-deshidratada-en-elmercado-de-los-estados-unidos-marzo-2018.

FAO (2010). Food and Agriculture Organization of the United Nations. Recuperado de: http://www.fao.org/ag/agn/pfl report en/ annexes/Annex4/Ecuador/Importancesummary.xls.

FAO (2011). Food and Agriculture Organization of the United Nations. Recuperado de: http://www.fao.org/3/a-i2697s.pdf.

Fischer, G., Almanza-Merchán, P. J., \& Miranda, D. (2014). Importancia y cultivo de la uvilla (Physalis peruviana L.). Revista Brasileira de Fruticultura, 36(1), 1-15. DOI: https://doi.org/10.1590/0100-2945-441/13.

Gereffi, G. (1994). Commodity Chains and Global Capitalism, Edition: Paperback, Chapter: 5, Publisher: Praeger Publishers, Editors: Gary Gereffi, Miguel Korzeniewicz, pp.95-122

Gereffi, G., \& Fernandez-Stark, K. (2016). Global Value Chain Analysis: A Primer 2nd Edition, Technical Report, Duke CGGC (Center on Globalization, Governance \& Competitiveness). Duke University. 
El sector hortofrutícola de Ecuador: Principales características socio-productivas de la red agroalimentaria de la uvilla (Physalis peruviana)

Gereffi, G., \& Stacey, F. (2009). Value Chain Governance: Briefing Paper. Recuperado de: https://www.marketlinks.org/sites/marketlinks.org/files/resource/files/vc governance briefing paper.pdf.

Glas Espinel, J., Alvarado Espinel, V., León Abad, S., \& Parra Fonseca, J. C. (2015). Ministerio de Industrias y Productividad. Recuperado de: http://www.industrias.gob.ec/wp-content/uploads/2017/01/politicalndustrialweb-16dic-16-baja.pdf.

Guerrero, D. (2012). La demanda de uvilla en el mercado alemán y la comercialización desde la parroquia de Piartal del cantón Montúfar provincia del Carchi. Tesis de grado, Universidad Estatal Politécnica del Carchi, Ecuador.

Herrera, P. (2015). Viabilidad de la producción y exportación de uvilla y sus derivados al mercado de Alemania. Tesis de grado, Pontificia Universidad Católica del Ecuador, Ecuador.

Herrera, R., \& Baquero, M. (2018). Las 5 fuerzas de Porter. Tesis de pregrado, Universidad de Chile, Chile.

Hilaca, D. (2017). Diseño de un Plan Estratégico para Exportar Uvilla Ecuatoriana a la Unión Europea. Tesis de Maestría, Universidad de Guayaquil, Ecuador.

Hinojosa, M., \& Ipiales, M. (2012). Estrategia de fortalecimiento de la cadena productiva de la uvilla como aporte al desarrollo de las zonas rurales de la provincia de Imbabura. Universidad Central del Ecuador, Ecuador.

Hirschman, A. (1959). The strategy of economic development. American Journal of Agricultural Economics., 41(2), 468469. DOI: https://doi.org/10.2307/1235188.

IFAD (2011). The International Fund for Agricultural Development. Recuperado de: https://www.ifad.org/documents/10180/f2136c2e-3b40-4074-9b71-bfac80efc410.

MAGAP (2017) Zonificación Agroecológica Económica del Cultivo de Uvilla En el Ecuador Continental, 2017. Recuperado de: http://fliphtml5.com/ijia/anhl/basic.

Mendoza, J., Rodríguez, A., \& Millán, P. (2012). Caracterización Físico Química de la Uchuva (Physalis peruviana) en la Región de Silvia Cauca. Revista Biotecnología en el Sector Agropecuario y Agroindustrial, 10(2), 188-196.

Miranda, L. (2011). Current State of Colombian Fruticulture and Perspectives for Its Development. Bras. Frutic., 199-205.

Montilla, F., Ceballos, R., Guerrero, A., Narváez, P., Romero, E., \& Villareal, M. (2016). Desarrollo rural con enfoque territorial DRET. Recuperado

de: http://www.oim.org.co/sites/default/files/Anexo\%20H \%20Ficha\%20de\%20proyecto\%20de\%20Uvilla.pdf.

Nuñez, V. M., Sánchez, E. P., Barrero, L. S., Mayorga, F. G., Gómez, M. R., Hernández, E. G., \& Lobo, M. (2014). Estado del arte de la invetigación en uvilla Physalis peruviana L. Corpoica, Bogotá

Orjuela Castro, J., Castañeda Calderón, C., \& Calderón, M. (2008). Análisis de la Cadena de valor en las estructuras productivas de uvilla y tomate de árbol en la Provincia de Sumapaz y el Distrito Capital. Revista científica y tecnológica de la facultad de ingeniería, 13 (2), 4-12.

Paez, C. (2018). El Régimen Impositivo a la Propiedad Agrícola en el Ecuador y los Perjuicios Socioeconómicos que Causó su Existencia. Universidad Central del Ecuador, Ecuador.

Proaño, O. (2003). Proyecto de factibilidad para la creación de una empresa productora y comercializadora de uvilla. Tesis de grado, Escuela Politécnica del Ejército, Ecuador.

PROECUADOR (2016). Boletín de Inteligencia de Mercados Agosto- Septiembre 2016, Recuperado de: https://issuu.com/pro-ecuador/docs/boletindic eneh.

PROECUADOR (2017). Boletín de Comercio Exterior 2017. Recuperado de: https://issuu.com/proecuador/docs/bce mayo.

Reyes-Beltrán, M., Guanilo-Reyes, C., Ibáñez-Cárdenas, M., García-Collao, C., Idrogo-Alfaro, J., \& Huamán-Saavedra, J. (2015). Efecto del consumo de Physalis peruviana L. (aguaymanto) sobre el perfil lipídico de pacientes con hipercolesterolemia. Acta méd. Peruana, 32(4), 195-201.

Scott, G. J. (2013). Adding Values to Value Chains. Revista de Administração de Empresas., 54(1), 67-79. DOI: https://doi.org/10.1590/S0034-759020140107.

SENPLADES (2018). Secretaría Nacional de Planificación y Desarrollo. Recuperado de: http://www.planificacion.gob.ec/zonas-distritos-y-circuitos/.

Sukhatme, P. (1956). Teoría de encuestas por muestreo con aplicaciones. México: Fondo de Cultura Económica.

Tobar, V. (2013). Estudio de factibilidad para la creación de una empresa productora y comercializadora de vino elaborado a base de uvilla (uvilla) en la ciudad de Quito. Tesis de grado, Universidad Politécnica Salesiana, Ecuador.

Zambrano, D. (2016). Cadena productiva de lácteos y su contribución al desarrollo rural del Ecuador. Estudio comparativo de la cadena láctea en el Cantón Riobamba y la experiencia de Galicia (España). Tesis de maestría, Universidad de Santiago de Compostela (USC), España.

Ciencia y Agricultura (Cien. Agri.) Vol. 16 (1). L-ISSN 0122-8420. elSSN 2539-0899.

Enero-Abril 2019, pp. 31-51. Tunja (Boyacá) - Colombia. DOI

https://doi.org/10.19053/01228420.v16.n1.2019.8809 